\title{
Power-Aware Position Vector Routing for Wireless Sensor Networks*
}

\author{
Sangsoo Lee ${ }^{1}$, Daeyoung Kim ${ }^{1}$, Sungjin $\mathrm{Ahn}^{1}$, and Noseong Park ${ }^{2}$ \\ ${ }^{1}$ Real-time and Embedded Systems Laboratory, \\ Information and Communications University (ICU), \\ 119 Munjiro, Yuseong-Gu, Daejeon, Korea, Postal Code: 305-714, \\ Phone: +82-42-866-6812, Fax: +82-42-866-6810 \\ \{sslee, kimd, sungjin\}@icu.ac.kr \\ ${ }^{2}$ Electronics and Telecommunications Research Institute (ETRI), \\ 161 Gajeong-Dong, Yuseong-Gu, Daejeon, Korea, Postal Code: 305-700 \\ Phone: +82-42-869-1735, Fax: +82-42-869-1711 \\ behackaetri.re.kr
}

\begin{abstract}
We propose PPVR, a power-aware position vector routing protocol for wireless sensor networks. PPVR is an energy efficient geographical routing protocol that improves existing geographic routing protocols in two ways. First, in the forwarding phase, PPVR reduces the transmission energy by passing packets through a relay node called a power-aware node. Second, PPVR provides an efficient void node avoidance scheme that mitigates the cases forwarding packets to a wrong path so that higher route discovery success rate is achieved. The simulation results shows that the PPVR outperforms existing geographic routing protocols including GPSR and GEAR, in terms of the energy consumption and the successful delivery rate.
\end{abstract}

\section{Introduction}

Wireless Sensor Network (WSN) is a network consisting of a number of tiny sensor nodes deployed in a region of interest, where each node is capable of processing and wireless communication. Because individual sensor node is small in size and memory, equipped sensors, the processing power and battery capability are limited, routing protocol developers must take care of all resource restrictions in order to provide the intended service. Of the restrictions, reducing energy consumption as much as possible is especially the most critical designing issue in developing a sensor network routing protocol.

As the location based applications $[7,10]$ in this field become more and more important and numerous methods to obtain the location information are developed in hardware (e.g., GPS) and software (e.g., distributed localization algorithms) ways [1, 8], routing protocols utilizing the geographical information are becoming popular [2, 3 , 9]. The major advantage of these geographic routing protocols including GPSR [2] and GEAR [3] is that it minimizes the overhead of maintaining routing information

* This research has been partially supported by ITRC project of Korea Ministry of Information and Communication. 
(mostly, routing tables) since it selects the next node to forward packets only depending on the location of the neighbor nodes. However, this characteristic of selecting the next node by local optimal view causes a critical problem called void area problem that is occurred when a routing path reaches to a node which has only one neighbor that has just sent a packet to it. This problem has to be well dealt with to provide high success rate of packet delivery.

In this paper, we present a power-aware position vector routing protocol (PPVR) which consists of the energy efficient packet forwarding mechanism and the void node avoidance scheme. In the forwarding phase, PPVR uses power-aware position vector routing that forwards packets through a power-aware node that makes the total transmission energy be reduced even if it increases the hop count to the destination. In addition, to avoid the cases for a packet to reach a void area, the PPVR utilizes gate nodes that have the information of void nodes so that it selects a path that does not lead to a void node area before reaching there. As a result, PPVR delivers control and data packets highly energy efficiently at a high success rate.

The rest of this paper is organized as follows. In the next section, power-aware position vector routing is presented, and in Section 3 the void node avoidance scheme is discussed. The performance evaluation results are shown in Section 4. Finally, Section 5 concludes this work.

\section{Power-Aware Position Vector Routing}

The position vector routing (PVR) is based on a greedy forwarding using the geographical information. Unlike the existing greedy forwarding algorithms used in GPSR and GEAR where a node selects the next node as a node that has the minimum distance to the destination node among its neighbors, position vector routing protocol uses two items of information to select the next node, the distance and the angle to the destination. The procedure determining the next node is as follows. First, a source node $O$ calculates the following function $f\left(N_{i}, D\right)$ for each neighbor node $N_{i}$ regarding to the location of the destination node $D$.

$$
f\left(N_{i}, D\right)=\left|N_{i}\right||D| \cos \left(A N G L E\left(N_{i}, D\right)\right)
$$

Here, $|X|$ denotes the distance from the source node $O$ to a node $X$ and $A N G L E(X$, $Y$ ) denotes the angle $\angle X O Y$. Then, the node which has the maximum value for the function $f\left(N_{i}, D\right)$ is selected as the next node to forward packets to the destination. Fig. 1 illustrates this procedure. By the definition of the function, as the distance is shorter and the angle is narrow, a node is likely to be selected as the next node.

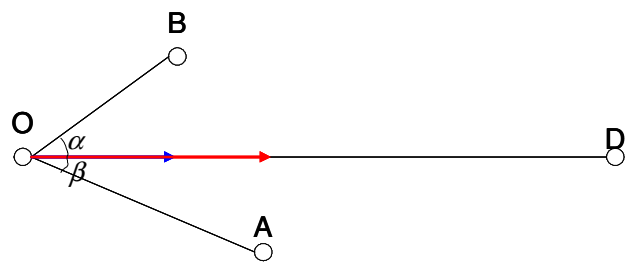

Fig. 1. Next node selection of PVR 


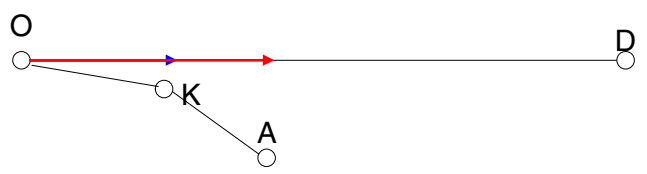

Fig. 2. Power-aware node selection of PPVR

The position vector routing introduced above however does not consider the energy efficiency sufficiently when it determines the next node. Power-aware position vector routing protocol (PPVR) improves the PVR with consideration of energy efficiency of senor networks.

The basic concept of the PPVR is that passing a packet by a neighbor node, called a relay node or a power-aware node, to a next node is more efficient in terms of transmission energy consumption even if the next node can be reached directly and passing by the relay node increases the hop count to the destination. Fig. 2 shows the case where passing a packet by a relay node $K$ is more efficient than transmitting it directly to the next node $A$. Now we present the procedure that a node finds the power-aware node for a next node in more detail.

When nodes are deployed, they first start to acquire the location information of its neighbor nodes. After this procedure, each node then attempts to acquire the poweraware nodes for all neighbors. The power-aware node selection function, which calculates the expected energy consumption, depends on what power consumption model is used. The most commonly used power consumption models are the well-known Friss free space model and two ray ground model. In these models, the transmission energy is proportional to the distance between the transmitter and the receiver. Exactly speaking, the consumed power $P(d) \propto 1 / d^{n}$, and $n=2$ and $n=4$ are used for Friss free space and two ray ground model, respectively [4].

According to the power consumption model, a node $O$ given a next node $A$ by the function $f\left(N_{i}, D\right)$ calculates the following power consumption function $f_{\text {power }}$ for all its neighbors $N_{i}$. V. Rodoplu et al [6] presented an assumption which is helpful to reduce the power consumption

$$
f_{\text {power }}\left(N_{i}\right)=d\left(O, N_{i}\right)^{n}+d\left(N_{i}, A\right)^{n}+2 \operatorname{recv}(n)
$$

This function denotes the expected power consumption when a node $O$ transmits a packet through a neighbor node $N_{i}$ to a next node $A$, and $d(X, Y)$ denotes the distance between nodes $X$ and $Y, \operatorname{recv}(n)$ denotes the energy consumption at the receiver. There are twice receptions at node $N_{i}$ and $A$. And the $n$ becomes 2 or 4 depending on the underlying power consumption model. Then, if expected power consumption through a power-aware node $N_{k}$ is less than that of directly sending to the next node $A$, the node $O$ selects the neighbor node $N_{k}$ as the power-aware node relaying packets to the next node $A$. If there is no node satisfying above condition, it directly sends packets to the next node $A$. The energy consumption of directly sending to $\mathrm{A}$ is:

$$
d(O, A)^{n}+\operatorname{recv}(n) .
$$

Therefore, the neighbor node satisfying the following condition and has the minimum power consumption becomes the power-aware node for the next node $A$. 


$$
d\left(O, N_{i}\right)^{n}+d\left(N_{i}, A\right)^{n}+\operatorname{recv}(n)<d(O, A)^{n}
$$

Fig. 3 shows this power-aware node selection procedure. Node $O$ is the source and $D$ is the destination, node $R, A$ and $N_{i}$ s are neighbors of node $O$, and dashed circle shows the communication range of node $O$. Node $A$ is selected as the next node for the destination node $D$ by node $O$ through the position vector routing presented previously and node $R$ indicates the power-aware node between nodes $O$ and $A$.

Figure 4 depicts the region where the power-aware node exists between $O$ and $A$. All the nodes in the power-aware region circle which includes node $\mathrm{R}$, satisfies the equation $d(O, R)^{2}+d(R, A)^{2}+\operatorname{recv}(n)<d(O, A)^{2}$, when $n=2$.
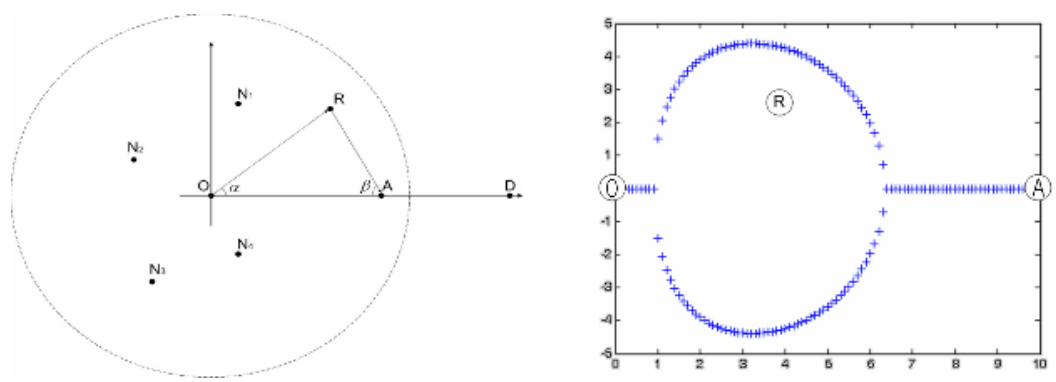

Fig. 3. Power-aware node selection Fig. 4. Power-aware region between node $O$ to node $A$

\section{Void Node Avoidance}

\subsection{Void Node Area Problem}

Geographical routing protocols select the next node using its local information. That is, it finds the solution that is optimal only in local. For example, GPSR selects the next node as the one which has the minimum distance to the destination, among its neighbors. However, even if it is locally optimal, it cannot guarantee the optimality in global. Thus, a routing path proceeded through the local optimal strategy may lead to extensively inefficient path or even route discovery failure, depending on the density and the topology of the network. Void node area is an area where a node does not have any neighbor to send a packet except the one from which it has just received the packet. When a route packet reaches to this area, it takes many steps to escape the area and then to find proper path toward the destination.

Fig. 5 shows an example of the void node area. Source node $S$ sends a route discovery request to the destination node $D$ using a geographical routing. When the packet reaches to the node $a_{2}$, it selects $b_{1}$ as the next node since it is the closest node to the destination among $a_{2}$ 's neighbors (here, $a_{3}$ ) and thus becomes the local optimal solution. However, it leads to the void node area since $b_{2}$ is a void node. Thus, it is better for $a_{2}$ to select $a_{3}$ instead of $b_{1}$ as the next node avoiding reaching to the void node.

GPSR and GEAR introduced solutions for this problem that escapes the void node area after reaching to the area. However, we here present an improved solution which selects the correct path avoiding entering to the void node area. 


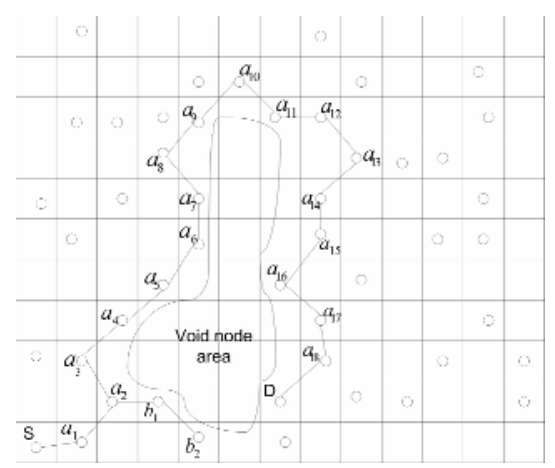

Fig. 5. Void node area problem

\subsection{Void Node Avoidance Scheme}

Void node is a node that has only one neighbor that has just sent a packet to it. Therefore, when a routing packet reaches to a void node, it cannot progress anymore. Gate node is a node that is the closest to the void node and having more than three neighbors. A set of connected nodes is said to be a node chain, if and only if a node $a_{k}$ in the chain has only two neighbors $a_{k-1}$ and $a_{k+1}$, except the two nodes at the ends of the chain, which are a gate node and a void node. A node chain is denoted as $a_{1} \leftrightarrow a_{2} \leftrightarrow \ldots \leftrightarrow a_{k} \leftrightarrow \ldots \leftrightarrow a_{n}$. For example, in Fig. 5, $b_{2}$ is a void node and $a_{2}$ is a gate node. Thus, the connected nodes, $a_{2} \leftrightarrow b_{1} \leftrightarrow b_{2}$, form a node chain. Now we present the detail void node avoidance algorithm.

After the acquisition of neighbor locations, a node can know whether it is a void node or not. If a node is turned out to be a void node, it sends a void region notification message to a gate node along its node chain. Then, a node receiving the notification message and has more than three neighbors becomes a gate node and there the notification ends. After void node acquisition and gate node selection procedure are completed, all gate nodes are aware of the void nodes and void area in their responsible territory. Thus, receiving a routing request packet, the gate node avoids guiding the packet toward the void node area by selecting the next node as the one that is not in any node chain. However, if the destination is in the middle of the node chain, this makes a problem.

So, a gate node has to keep the information of the nodes in the chain. In some worse cases where the density of the network is low, the length of the node chain may become larger and this means that the amount of information a gate node should keep becomes larger as well. PPVR presents a mechanism that only keeps the information at most a half of the total number of nodes in a node chain by the following theorem.

Theorem 1: Given a node chain, $a_{1} \leftrightarrow a_{2} \leftrightarrow \ldots \leftrightarrow a_{k-1} \leftrightarrow a_{k}$, the gate node $a_{1}$ only requires floor $(k / 2)$ number of nodes information to discover all the nodes in the chain, where $k$ is the number of nodes in the chain.

Proof: By the definition of the node chain, a node $a_{i}$ has only two neighbors $a_{i-1}$ and $a_{i+1}$, and the two neighbor nodes cannot communicate with one another. Thus, as shown in Fig. 6, given the locations of two nodes $A$ and $C$, and that they are out of 
communication range $r$ each other, but share a node, it can be inferred that there is only one node (here $B$ ) between the intersection range of $A$ and $C$. This means that it does not require knowing the location information of node $B$ when we know the location of $A$ and $C$.
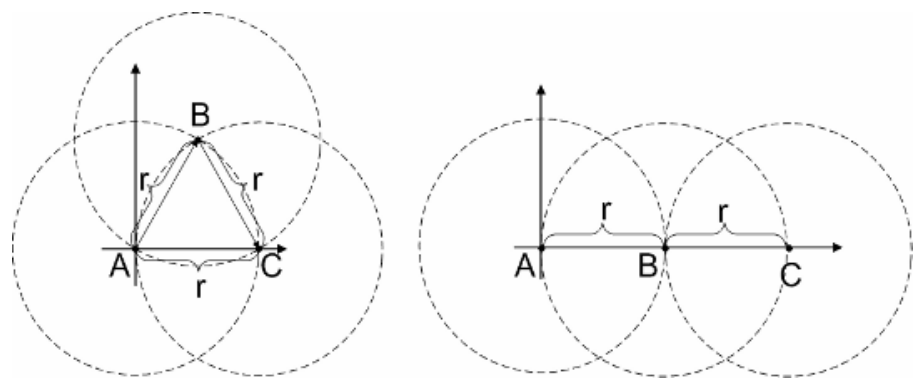

Fig. 6. Reducing location information to $\operatorname{lloor}(k / 2)$

Now, we compare how PPVR works with the void node avoidance scheme to without one.

\section{Without void node avoidance scheme}

In figure 7 , assuming that $V_{l}$ is the source and $C$ is the destination. $V_{l}$ chooses $m_{11}$ as next node by position vector routing, then $m_{11}$ selects $G_{l}, G_{1}$ select $m_{21}$ as the next node by the local optimal strategy and then $m_{21}$ according to position vector routing, and it finally reaches to $V_{2}$ the void node. This leads to route discovery failure if proper void area avoidance mechanism is not provided even though there are alternative path going to $G_{2}$ at $G_{l}$.

\section{With void node avoidance scheme}

If the void node avoidance scheme is supported, when it reaches to $G_{1}$, it selects $G_{2}$ as the next node since $G_{l}$ is aware that going through $m_{2 l}$ leads to a void node and that

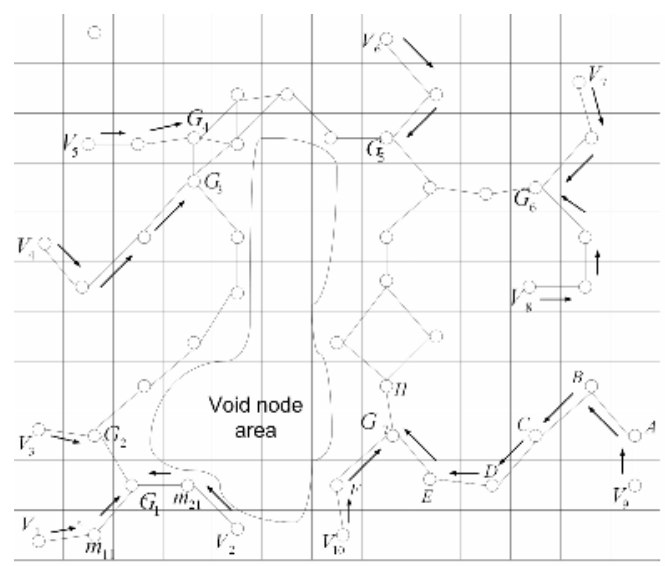

Fig. 7. PPVR with void node area avoidance scheme 
the destination is not in the node chain. Then, following the same procedure, it finally reaches to $G_{7}$ by the power-aware position vector routing. At $G_{7}$, it selects $E$ as the next node even if it is a node chain consequently leading to a void node, since $G_{7}$ is keeping the location information of $V_{9}, B$, and $D$, and from this information it can infer that the destination is in the middle of $D$ and $B$ as explained in above theorem.

\section{Performance Evaluation}

\subsection{Simulation Environment}

The MICA2 [5] uses CSMA/CA MAC and $433 \mathrm{MHz}$ RF chip that gives the maximum output power of $10 \mathrm{dBm}$ with the sensitivity of $-109 \mathrm{dBm}$. It consumes $5.3 \mathrm{~mA}$ and $26.7 \mathrm{~mA}$ at its minimum and maximum signal strengths, respectively, and $7.4 \mathrm{~mA}$ in receiving mode. It can send at most 53 packets in a second with the packet size of 42 bytes. We assume that the antenna is omni-directional with $1 \mathrm{~dB}$ gain and located $10 \mathrm{~cm}$ above the ground. The maximum transmission range becomes $10 \mathrm{~m}$ by the specification. Two-ray model [4] is used for power consumption estimation. In a 50 $\mathrm{m} \times 50 \mathrm{~m}$ sensor field, 200 sensor nodes are randomly deployed, for the evaluation of the local minimum problem. $10 \mathrm{~m} \times 10 \mathrm{~m}$ void node area is deployed randomly in the sensor field.

For the simplicity of simulation, each sensor node has $4 \mathrm{~J}$ as its initial energy and sends data packets in a period ranging from 1 to 10 seconds (this is randomly chosen for each node). Considering the sensor node's restricted memory and poor communication conditions, the packet retransmission is not allowed, if the MAC layer fails to transmit any packet in a node. We compare the PPVR and PVR algorithms with the GEAR and GPSR algorithms.

\subsection{Evaluation of Power Aware Node and Void Node Avoidance Scheme}

We have evaluated our proposed idea in two different criteria. First, considering the energy consumption aspect, it shows how well our scheme saves energy as compared to the two existing schemes, GPSR and GEAR. Second, considering the ability avoiding the local minimum problem, it proves that how short the hop count would be and how the proposed avoidance scheme is effective for sensor networks.

PVR works basically the similar way to the GPSR, except for avoidance of local minimum. However, PPVR additionally uses the relay nodes, it can reduce more energy in packet transmission resulting in more alive nodes. In Fig. 8(a), PPVR and PVR prove that they can save larger amount of energy than others can do. Thanks to the transmission power control, when it is applied to the sensor network, it shows much saving of energy as PVR does. Furthermore, when power-aware node scheme is applied, PPVR outperforms GPSR and GEAR, and PVR as well. This means that as the power aware nodes are added in the initial PVR route, it further saves energy. Here, GPSR is the worst case due to the lack of energy saving facility.

When PPVR and PVR are applied, the number of nodes exhausting energy is smaller than the others. It results in the longevity of the sensor network. Now, we examine the average remaining energy of the sensor network with respect to time $t$. Fig. 8(b) shows PPVR and PVR guarantee higher average remaining energy. 


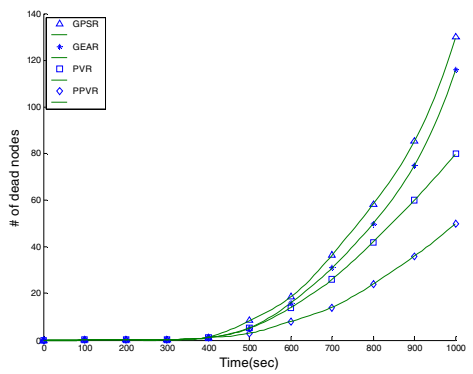

(a) Energy exhausted nodes by time $\mathrm{t}$

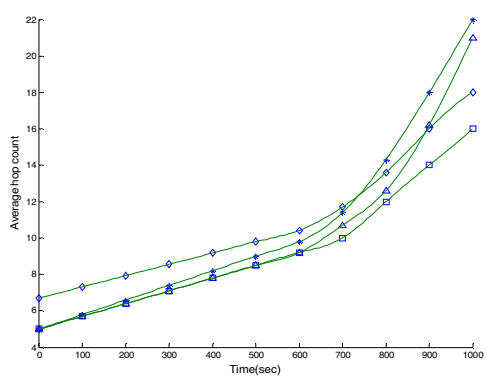

(c) Average hop count (1)

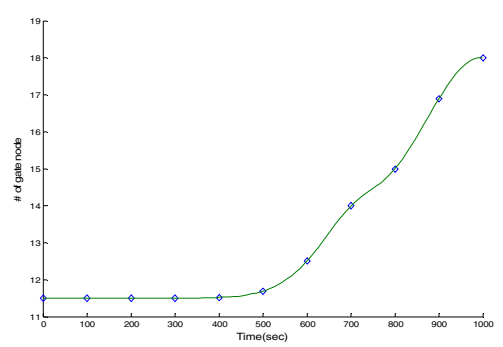

(e) Number of gate nodes by time $t$

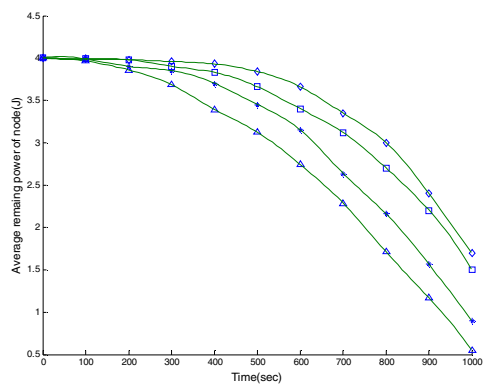

(b) remaining energy of nodes by time

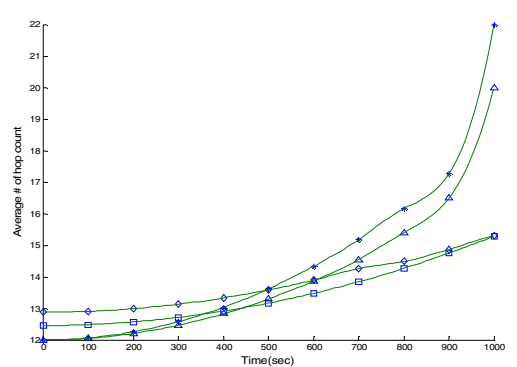

(d) Average hop count (2)

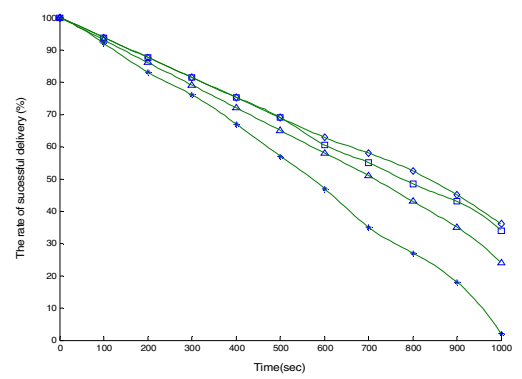

(f) Successful packet transmission rate

Fig. 8. Simulation results: $50 \mathrm{~m}$ x 50m sensor field with 200 nodes deployed randomly

Both Fig. 8(a) and 8(b) explain that PPVR is superior to others in reduction of energy consumption. However, the superiority must pay back with additional number of hop counts.

In Fig. 8(d), we can see the drawback of PPVR. It proves that the average number of hop counts of PPVR is greater than the other schemes in earlier time period. But, over its last lifetime, PPVR shows smaller hop counts than the others due to the void node avoidance scheme.

For more intensive evaluation of void node avoidance scheme, we simulated all the schemes deploying a $10 \mathrm{~m}$ X $10 \mathrm{~m}$ void node area randomly. In Fig. 8(d), in the early stage of the simulation, the average hop counts of PPVR is larger than the others. It 
results from the effect of the power aware node. As regards the PVR, we can easily tell that its average hop count (which doesn't include the power aware node) is slightly less than GEAR and GPSR. However, PPVR becomes better as the number of packet transmission increases, thanks to the void node avoidance scheme. Thus, for overcoming the local minimum, void node avoidance scheme is more effective than the learning based avoidance by GEAR and the right hand rule by GPSR. To accomplish this significant performance improvement, we use the gate node approach. Fig. 8(e) shows the increase of the number of gate nodes with respect to time $t$. It is necessary for PPVR to have void node avoidance scheme.

When the void node avoidance scheme (which is the avoidance of local minimum) is applied and the power aware node is included to further save energy, there are two notable improvements - firstly, the longevity of the sensor network (which was proved by the early simulation result) and secondly, the improvement in successful routing.

In Fig. 8(f), it explains that PPVR and PVR outperform others in terms of the number of successful packet delivery rate (thanks to the void node avoidance scheme and power aware node).

\section{Conclusion}

In the paper, we propose a power-aware geographical routing protocol PPVR for sensor networks (which minimizes the energy consumption and results in the longevity of sensor networks) and local minimum avoidance scheme (which leverages successful packet delivery rate and minimizes average hop count considerably). The simulation results prove that PPVR improves the energy efficiency and packet delivery success rate compared to the existing geographical routing protocols including GPSR and GEAR.

\section{References}

1. J. Hightower and G. Bordello. Location systems for ubiquitous computing. In IEEE Comp., 2001.

2. B. Karp and H. T. Hung, GPSR: Greedy Perimeter Stateless Routing for Wireless Networks, In ACM MOBICOM, 2000.

3. Y. Yu, et al. Geographical and Energy Aware Routing: a recursive data dissemination protocol for wireless sensor networks. In UCLA CS Tech. Report, 2001.

4. J. D. Kraus and R. J. Marhefka, Third edition: Antennas for all applications, Mc Graw Hill.

5. Crossbow Technology Inc. http://www.xbow.com

6. V. Rodoplu and T.H. Meng, Minimum Energy Mobile Wireless Networks, IEEE J. Sel. Areas in Comm. (JSAC), 1999.

7. C. Intanagonwiwat, R. Govindan and D. Estrin, Directed diffusion: A scalable and robust communication paradigm for sensor networks, In ACM MOBICOM, 2000.

8. N. Bulusu, J. Heidemann, and D. Estrin, GPS-Less Low Cost Outdoor Localization for Very Small Devices, IEEE Personal Comm. Magazine, 2000.

9. Y.B. Ko and N.H. Vaidya, Location aided routing (LAR) in mobile ad-hoc networks, In ACM MOBICOM, 1998.

10. A. Cerpa, J. Elson, D. Estrin, L. Girod, M. Hamilton, and J. Zhao. Habitat monitoring: Application driver for wireless communications technology. In ACM SIGCOMM Workshop on Data Communications in Latin America and the Caribbean, 2001. 